\title{
Numerical simulations of self-focusing of ultrafast laser pulses
}

\author{
Gadi Fibich* \\ School of Mathematical Sciences, Tel Aviv University, Tel Aviv 69978, Israel \\ Weiqing Ren \\ Courant Institute of Mathematical Science, New York University, New York, New York 10012 \\ Xiao-Ping Wang \\ Department of Mathematics, The Hong Kong University of Science and Technology, Clear Water Bay, Kowloon, Hong Kong
}

(Received 14 November 2002; published 7 May 2003)

\begin{abstract}
Simulation of nonlinear propagation of intense ultrafast laser pulses is a hard problem, because of the steep spatial gradients and the temporal shocks that form during the propagation. In this study we adapt the iterative grid distribution method of Ren and Wang [J. Comput. Phys. 159, 246 (2000)] to solve the two-dimensional nonlinear Schrödinger equation with normal time dispersion, space-time focusing, and self-steepening. Our simulations show that, after the asymmetric temporal pulse splitting, the rear peak self-focuses faster than the front one. As a result, the collapse of the rear peak is arrested before that of the front peak. Unlike what has sometimes been conjectured, however, collapse of the two peaks is not arrested through multiple splittings, but rather through temporal dispersion.
\end{abstract}

DOI: 10.1103/PhysRevE.67.056603

PACS number(s): 42.25.Bs, 42.65.Sf, 42.65.Jx

\section{INTRODUCTION}

The nonlinear Schrödinger equation (NLS)

$$
i \psi_{z}(z, x, y)+\Delta_{\perp} \psi+|\psi|^{2} \psi=0
$$

is the model equation for the propagation of $\mathrm{cw}$ (continuous wave) laser beams in Kerr media. Here, $\psi$ is the electric field envelope, $z$ is the axial distance in the direction of beam propagation, $x$ and $y$ are the coordinates in the transverse plane, and $\Delta_{\perp}=\partial_{x x}+\partial_{y y}$ is the diffraction term. It is well known that when the power, or $L^{2}$ norm, of the input beam is sufficiently high, solutions of Eq. (1) can self-focus and become singular in a finite distance $z[1,2]$. Because of the infinitely large gradients that exist at the singularity, standard numerical methods break down after the solution undergoes moderate focusing [3]. Therefore, as part of the research effort during the 1980s to find the blowup rate of the NLS, McLaughlin et al. developed the numerical method of $d y$ namical rescaling [4], which can resolve the solution near the singularity at extremely high amplitudes. This method exploits the known self-similar structure of the collapsing part of the solution near the singularity, which relates the shrinking transverse width of the solution to the increase in its norm. Therefore, the solution is computed on a fixed computational grid, which in physical space corresponds to a grid that shrinks uniformly toward the singularity. The focusing rate of the grid points is determined dynamically from some norm of the solution $\left(\left.\int|\nabla| \psi\right|^{2} d x d y\right.$, $\max _{x, y}|\psi|$, etc.). Because the focusing rate of the grid points can be chosen to be the same as the physical focusing rate, in the transformed variables the solution remains smooth and can thus be solved using "standard" methods.

\footnotetext{
*Electronic address: fibich@math.tau.ac.il
}

The method of dynamic rescaling works extremely well for solutions of the NLS with radially symmetric initial conditions, in which case focusing by $10^{10}$ or more can easily be realized (see, e.g., Fig. 3.5 in [1]). Although the method of dynamic rescaling has been extended to NLS's with nonisotropic initial conditions [5] and to perturbed NLS's (e.g., NLS's with normal time dispersion [6]), in such cases dynamic rescaling is considerably less efficient, because the solution does not focus uniformly and/or it is not clear how to extract the physical focusing rate from the solution. The iterative grid redistribution (IGR) method, developed by Ren and Wang, overcomes these difficulties by allowing the grid points to move independently (rather than uniformly) according to a general variational minimization principle. This method has been showed to be highly effective for solving partial differential equations (PDE's) with singular behavior such as the NLS (1) and the Keller-Segal equations with multiple blowup points [7]. As we shall see, however, applying the IGR method to nonstationary NLS models that describe the propagation of ultrashort pulses turns out to be considerably more demanding.

\section{A. Simulations of ultrashort pulses}

The NLS (1) does not include temporal effects. These effects become important in the case of ultrashort laser pulses, whose propagation can be modeled by the dimensionless nonstationary NLS [8]

$$
\begin{aligned}
& i \psi_{z}(z, x, y, t)+\Delta_{\perp} \psi+|\psi|^{2} \psi+\epsilon_{1} \psi_{z z} \\
& \quad+i \epsilon_{2}\left[\left(|\psi|^{2} \psi\right)_{t}-\Delta_{\perp} \psi_{t}\right]-\epsilon_{3} \psi_{t t}=0
\end{aligned}
$$

where now $\psi$ is also a function of time $t$. The dimensionless parameters are given by 


$$
\epsilon_{1}=\frac{1}{4 r_{0}^{2} k_{0}^{2}}, \quad \epsilon_{2}=\frac{1}{c_{g} k_{0} T} \frac{c}{n_{0} c_{g}}, \quad \epsilon_{3}=\frac{L_{\mathrm{diff}} k_{\omega \omega}}{T^{2}}
$$

where $r_{0}$ and $T$ are the transverse width and temporal duration of the input pulse, respectively, $k=\omega n_{0}(\omega) / c, k_{0}$ $=k\left(\omega_{0}\right), c_{g}^{-1}=(d k / d \omega)_{\omega_{0}}$, and $L_{\text {diff }}=r_{0}^{2} k_{0}$ is the diffraction length. In the case of ultrashort laser pulses $\epsilon_{1}$ is considerably smaller than $\epsilon_{2}$ and $\epsilon_{3}$ [8]. Therefore it is reasonable to neglect the nonparaxial term $\epsilon_{1} \psi_{z z}$, in which case Eq. (2) reduces to

$$
\begin{aligned}
& i \psi_{z}(z, x, y, t)+\Delta_{\perp} \psi+|\psi|^{2} \psi \\
& \quad+i \epsilon_{2}\left[\left(|\psi|^{2} \psi\right)_{t}-\Delta_{\perp} \psi_{t}\right]-\epsilon_{3} \psi_{t t}=0 .
\end{aligned}
$$

The scalar equation (2) does not take into account the vectorial nature of the electric field. Since the magnitude of vectorial effects is also $O\left(\epsilon_{1}\right)$ [9-11], neglecting vectorial effects is justified within the framework of Eq. (3).

Self-focusing in the two-dimensional (2D) NLS, Eq. (1), is highly sensitive to small perturbations. A general method for analyzing the effect of small perturbations in the $2 \mathrm{D}$ NLS, called modulation theory, was developed in $[1,12]$. Application of modulation theory to Eq. (2) showed that the self-focusing pulse would undergo an asymmetric temporal splitting [8]. Such asymmetric splitting was later observed experimentally, as well as in numerical simulations of perturbed NLS equations that are equivalent to Eq. (3) (see [1315]). After asymmetric temporal splitting the solution develops temporal shocks. As a result, standard numerical methods break down shortly after the pulse splitting. In order to find the postsplitting dynamics we decided to solve Eq. (3) using the IGR method. It turned out that solving Eq. (3) is considerably more demanding than solving the stationary NLS, because in addition to the extra dimension and the high resolution near the two peaks, one needs to have sufficient global resolution for resolving the temporal oscillations that develop over the whole domain. As a result, although solving
Eq. (3) with the "original" IGR method improved on previous simulation results, these simulations could still not proceed much past the temporal splitting. Therefore, in this study we improved the IGR method by introducing a highorder finite-difference scheme on nonuniform grids. This improvement enabled us to present numerical simulations of ultrashort pulse propagation well beyond the asymmetric temporal splitting.

\section{B. Organization}

The paper is organized as follows. In Sec. II we review the original IGR method, which was introduced in [7]. In Sec. III we describe two accuracy improvements that are needed for resolving shocklike structures and strong oscillations in the nonstationary NLS: the use of a high-order scheme on a nonuniform grid, and of an orthogonal grid. In Sec. IV we use the improved IGR method to solve the timedispersive NLS

$$
i \psi_{z}(z, x, y, t)+\Delta_{\perp} \psi-\epsilon_{3} \psi_{t t}+|\psi|^{2} \psi=0 .
$$

We begin with Eq. (4), since most research on propagation of ultrashort pulses has been carried out within the framework of this equation. In addition, for many years the postsplitting dynamics remained a matter of debate, since "standard" numerical methods broke down shortly after the splitting. Indeed, the first reliable simulations of Eq. (4) which could go beyond the pulse splitting were obtained only recently in [16]. The results obtained using the IGR method agree qualitatively with those of [16], which were obtained using a different numerical method, thus providing support for the validity of the results obtained with these two methods. We also add to the results of [16] by showing that temporal dispersion becomes the dominant mechanism after the pulse splitting and therefore that the arrest of collapse of the two peaks is predominantly a linear time-dispersion effect. Finally, we present simulations with the initial conditions used by Zharova et al. [17], which go beyond the pulse splitting. Our
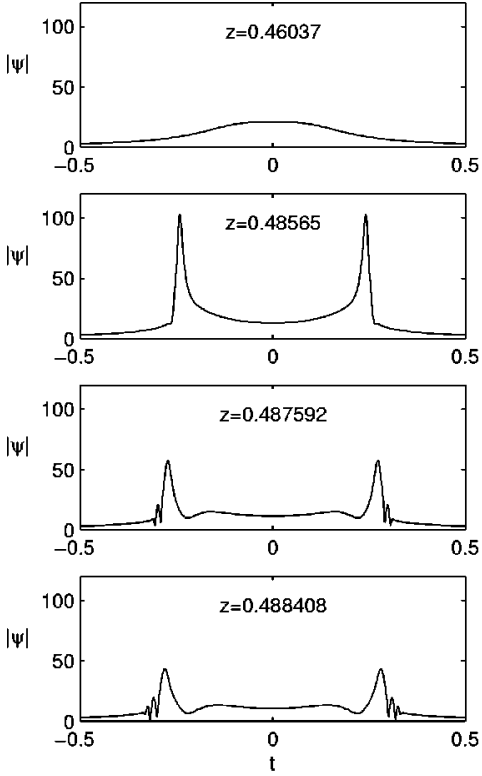
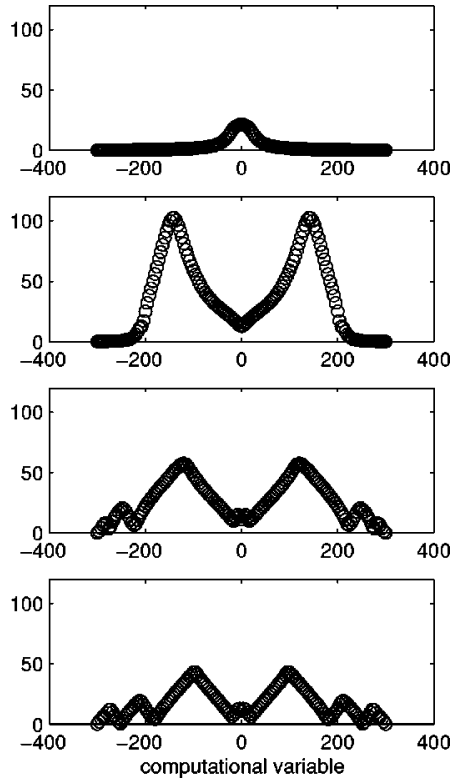

FIG. 1. On-axis amplitude $|\psi(z, r=0, t)|$ for the solution of Eq. (4) with the initial condition (10) and $A=1.75$. The right column shows the corresponding solution in the computational variable of $t$ (only one-fourth of the grid points are shown). 

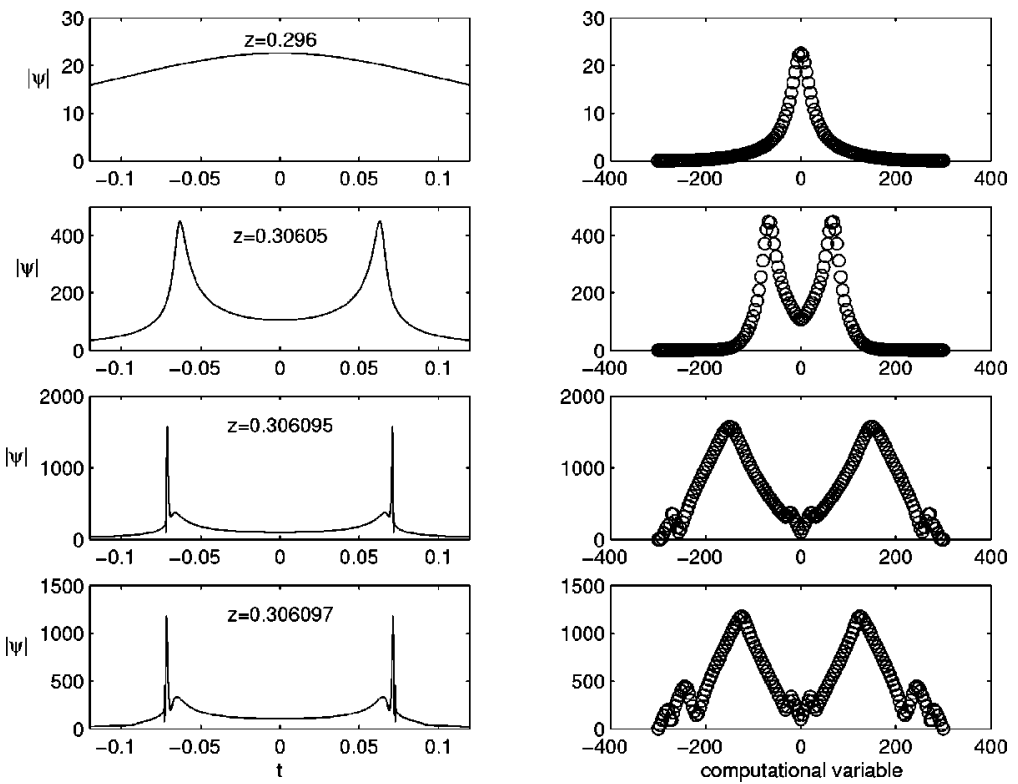

FIG. 2. Same as Fig. 1 with $A=2$.

simulations show that the secondary splittings observed in [17] were a numerical artifact.

In Sec. V we apply the improved IGR method to solve the full model equation (3). Our results confirm the observation made in [13] that after the asymmetric pulse splitting there is a reversal in the relative heights of the two peaks. Using the IGR method, however, we were able to continue the numerical integration even further, and therefore to observe the arrest of collapse in Eq. (3). As in the case of Eq. (4), temporal dispersion becomes the dominant mechanism after the pulse splitting, and the arrest of the collapse of the two peaks is predominantly a linear time-dispersion effect.

\section{ADAPTIVE METHOD BASED ON THE ITERATIVE GRID REDISTRIBUTION}

We now briefly review the adaptive mesh method, which is based on an iterative grid generation procedure [7].

\section{A. Grid distribution based on the variational principle}

In two (or higher) spatial dimensions, mesh distribution is usually obtained using a variational approach, specifically by minimizing a functional of the coordinate mapping between the physical domain and the computational domain. The functional is chosen so that the minimum is suitably influenced by the desired properties of the solution $u(\mathbf{x})$ of the PDE itself.

Let $\mathbf{x}$ and $\xi$ denote the physical and computational coordinates, respectively, on the computational domain $\Omega \in \mathbf{R}^{d}$. A one-to-one coordinate transformation on $\Omega$ is denoted by

$$
\mathbf{x}=\mathbf{x}(\xi), \quad \xi \in \Omega .
$$

The functionals used in existing variational approaches for mesh generation and adaptation can usually be expressed in the form
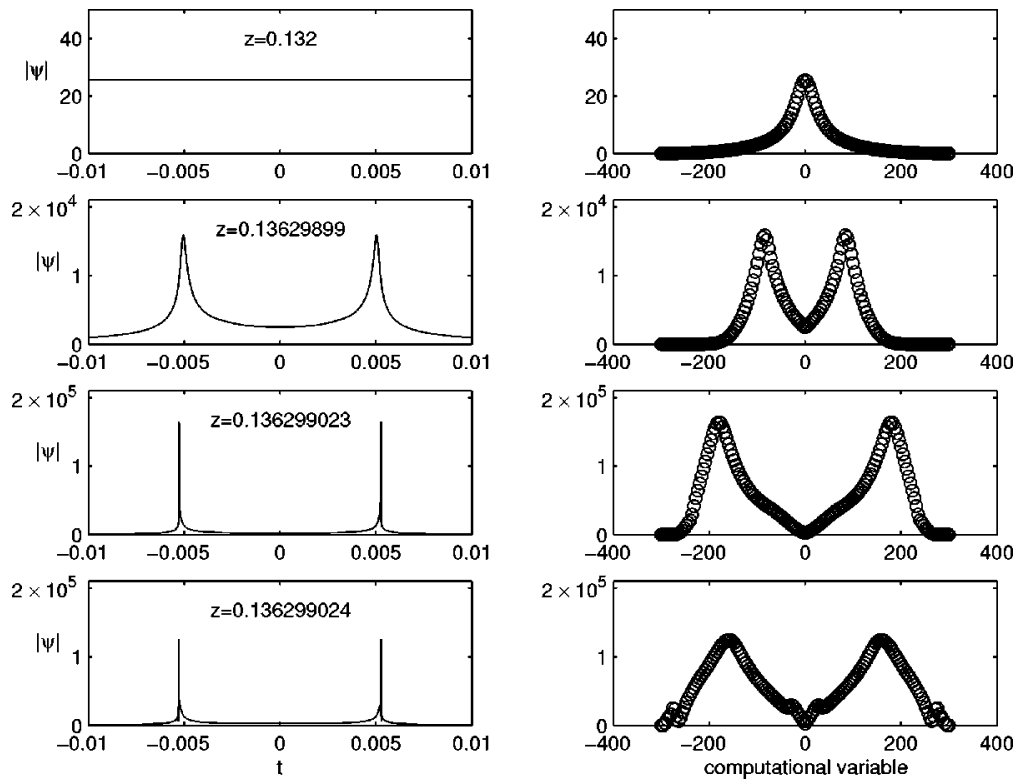

FIG. 3. Same as Fig. 1 with $A=3$. 

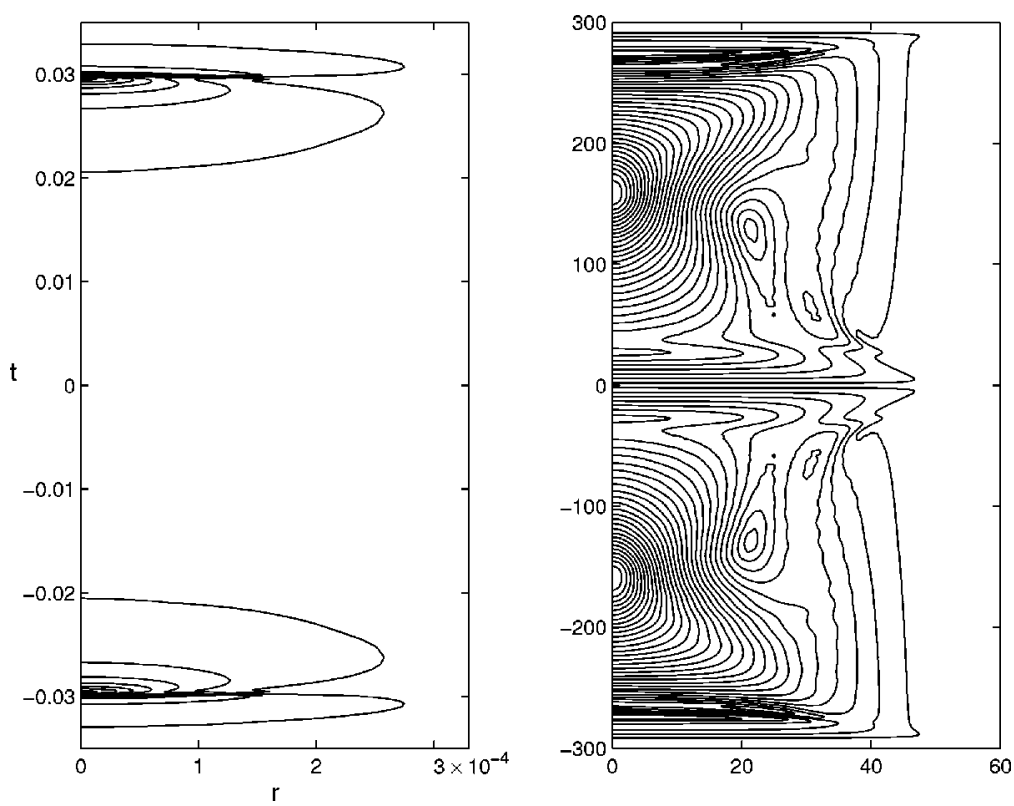

FIG. 4. Contour plot of $|\psi(z=0.136299024, r, t)|$ in the simulation of Fig. 3, in physical variables (left) and computational variables (right).

$$
E(\xi)=\int_{\Omega} \sum_{i, j, \alpha, \beta} g^{i, j} \frac{\partial \xi^{\alpha}}{\partial x^{i}} \frac{\partial \xi^{\beta}}{\partial x^{j}} d \mathbf{x},
$$

where $G=\left(g_{i, j}\right), G^{-1}=\left(g^{i, j}\right)$ are symmetric positive definite matrices that are monitor functions in a matrix form. Normally, we choose $g_{i, j}=\delta_{i, j}+u_{x_{i}} u_{x_{j}}$. The coordinate transformation and the mesh are determined from the EulerLagrange equation

$$
\boldsymbol{\nabla} \cdot\left(G^{-1} \nabla \boldsymbol{\xi}\right)=0
$$

We note that more terms can be added to the functional (6) to control other properties of the mesh, such as orthogonality of the mesh and the alignment of the mesh lines with a prescribed vector field [18].
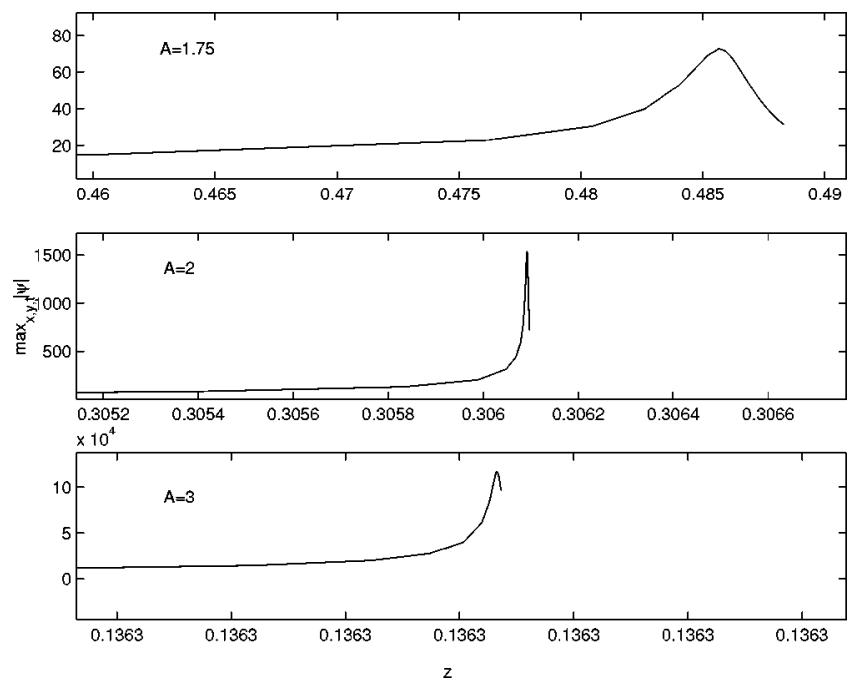

FIG. 5. $\max _{x, y, t}|\psi|$ as a function of $z$, for the simulations of Figs. $1-3$.

\section{B. An iterative grid redistribution procedure}

Let us first define the grid mapping:

$$
\mathbf{T}: \quad(\mathbf{x}, \quad u(\mathbf{x})) \rightarrow(\xi, \quad v(\xi))=(\xi, u(\mathbf{x}(\xi))) .
$$

Here $\mathbf{x}=\mathbf{x}(\xi)$ is determined from Eq. (7) with a monitor matrix involving $u(\mathbf{x})$.

If the monitor matrix $G$ is properly chosen, the resulting mesh should concentrate more grid points in the regions with large variations. This also means that $v(\xi)$ should be better behaved than the original function $u(\mathbf{x})$ in the sense that the variation of the monitor function in the new variables is reduced. However, in [7], we show that in some cases the resulting improvement is very limited. A natural idea for further improvement is to repeat the same procedure for $v(\xi)$. In fact, this process can be repeated until a satisfactory $v(\xi)$ is achieved. Based on this intuition, an iterative grid redistribution procedure is introduced by applying the grid mapping T iteratively: (1) Let $u^{k}(\mathbf{x})$ be the function after $k$ iterations; (2) determine the mapping $\mathbf{x}^{k+1}(\xi)$ from $u^{k}(\mathbf{x})$ according to Eq. (7) where the monitor matrix $w^{k}$ is defined using $u^{k}(x)$; and (3) define $u^{k+1}(\xi):=u^{k}(\mathbf{x}(\xi))$.

For example, after two iterations, we have

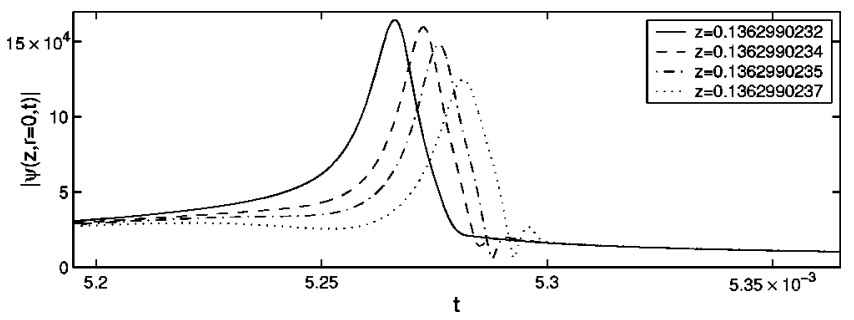

FIG. 6. Enlarged view of $|\psi(z, r=0, t)|$ in the simulation of Fig. 3 , showing decay of maximum amplitude and emergence of small structures. 

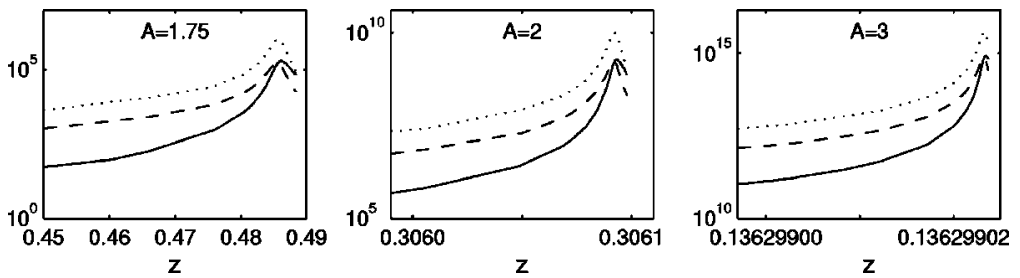

FIG. 7. $\max _{x, y, t}\left|\epsilon_{3} \psi_{t t}\right|$ (solid), $\max _{x, y, t}\left|\Delta_{\perp} \psi\right|$ (dotted), and $\left.\max _{x, y, t}\left|\Delta_{\perp} \psi+\right| \psi\right|^{2} \psi \mid$ (dashed) as a function of $z$, for the simulations of Figs. 1-3. $(\mathbf{x}, u(\mathbf{x})) \rightarrow^{T}\left(\xi_{1}, v_{1}\left(\xi_{1}\right)\right) \rightarrow^{\mathbf{T}}\left(\xi_{2}, v_{2}\left(\xi_{2}\right)\right)$.

In the first iteration, we determine a grid mapping $\mathbf{x}\left(\xi_{1}\right)$ and $v_{1}\left(\xi_{1}\right)=u\left(\mathbf{x}\left(\xi_{1}\right)\right)$. In the second iteration, we have $\xi_{1}\left(\xi_{2}\right)$ and $v_{2}\left(\xi_{2}\right)=v_{1}\left(\xi_{1}\left(\xi_{2}\right)\right)$

The result of the iteration is to flatten out the monitor function gradually. In fact, if $u^{k}(\mathbf{x})$ and $\mathbf{x}^{k}(\xi)$ converge, then we must have $\mathbf{x}^{k} \rightarrow \mathbf{x}^{*}(\xi)=\xi$ and $u^{k} \rightarrow u^{*}(\mathbf{x})$.

\section{Adaptive procedure for solving nonlinear Schrödinger equations}

We now incorporate the iterative remeshing into a static adaptive method for solving nonstationary NLS equations whose solution is $\psi=\psi(r, t, z)$. Recall that these are initial value problems in $z$, in which $t$ plays the role of a third spatial variable.

The procedure is as follows.

(1) Given an initial condition $\psi(r, t, z=0)$, the initial grid transforms $r(\rho, \tau), t(\rho, \tau)$ are determined from the iterative remeshing, which in turn gives an initial condition in the computational domain $\psi(r(\rho, \tau), t(\rho, \tau), z=0)$. The solution $\psi\left(\rho, \tau, z^{*}\right)$ cannot meet a certain smoothness criterion.

(2) Generate a new mesh by iterative remeshing, starting with $\psi\left(\rho, \tau, z^{*}\right)$. The remeshing iteration stops if the criterion in (1) is satisfied. Interpolation is used to generate the solution at the new grid points.

(3) Go to (1) to continue the integration.

\section{HIGH-ORDER SCHEME ON ORTHOGONAL GRIDS}

Solving Eq. (3) turns out to be considerably more demanding than solving the stationary NLS (1), because in addition to the extra dimension and the high resolution near the focal points, one also needs to resolve global oscillation as well as shocklike temporal structures. To overcome these difficulties we introduce two accuracy improvements: the use of a high-accuracy, modified central difference scheme and of a globally orthogonal grid.

\section{A. High-accuracy finite-difference scheme on nonuniform gird}

It is easy to see that the usual central difference (CD) scheme has lower accuracy on the nonuniform grid than that on the uniform grid. Denote the mesh size $h_{i}=x_{i}-x_{i-1}$. From Taylor expansion, we have

$$
\begin{aligned}
u^{\prime}\left(x_{i}\right)= & \frac{u\left(x_{i+1}\right)-u\left(x_{i-1}\right)}{h_{i}+h_{i+1}}-u^{\prime \prime}\left(x_{i}\right)\left(h_{i+1}-h_{i}\right) / 2-u^{\prime \prime \prime}\left(x_{i}\right) \\
& \times\left(h_{i+1}^{2}-h_{i+1} h_{i}+h_{i}^{2}\right) / 6 .
\end{aligned}
$$

In general, $h_{i+1} \neq h_{i}$ on a nonuniform grid. Therefore, the scheme has a similar accuracy as a first order scheme. However, we can obtain a more accurate scheme with the following difference formula:

$$
\begin{aligned}
u^{\prime}\left(x_{i}\right)= & {\left[a u\left(x_{i+1}\right)+b u\left(x_{i-1}\right)-(a+b) u\left(x_{i}\right)\right] } \\
& -u^{\prime \prime \prime}\left(x_{i}\right) h_{i} h_{i+1} / 6,
\end{aligned}
$$

where $a=1 / h_{i+1}-1 /\left(h_{i}+h_{i+1}\right)$ and $b=1 /\left(h_{i}+h_{i+1}\right)-1 / h_{i}$. Obviously, this is a more accurate scheme than Eq. (8) since Eq. (9) does not have an $O\left(h_{i}\right)$ order term any more. We call this the modified central difference (MCD) scheme. On the uniform grid, the MCD scheme is the same as the CD scheme. But the MCD scheme is much more accurate than the $\mathrm{CD}$ scheme on the nonuniform grid.

\section{B. Orthogonal grid}

Orthogonal grids offer significant advantages in numerical simulations of PDE's. In general, the accuracy of the finitedifference schemes is highest on orthogonal grids. The highaccuracy finite-difference scheme introduced in the previous section can be easily implemented on the orthogonal and rectangular grid. The orthogonal grid can be achieved by choosing the monitor matrix to be diagonal; each diagonal element depends on one variable only. Since our domain is rectangular, effectively this means that we obtain the twodimensional grid as a product of two one-dimensional grids. With the implementation of the high-accuracy scheme, we are able to compute solutions with much higher accuracy, which is significant in our simulations.

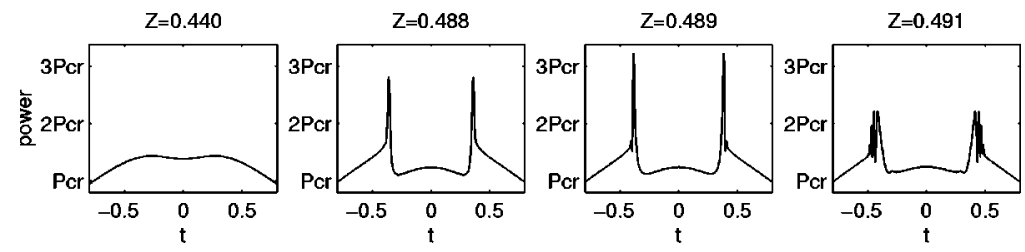

FIG. 8. Cross-sectional power $\int|\psi(z, x, y, t)|^{2} d x d y$, for the simulation of Fig. 1 . 

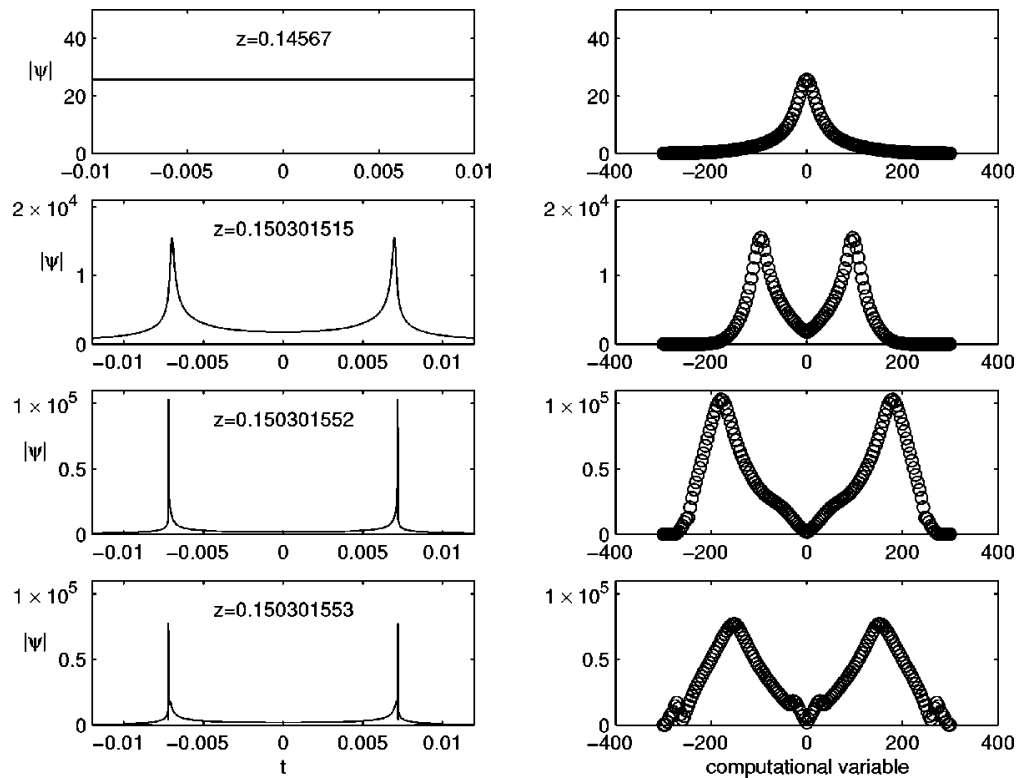

\section{TIME-DISPERSIVE NLS}

Until recently most studies of propagation of ultrashort pulses considered the time dispersive NLS model (4). When time dispersion is anomalous $\left(\epsilon_{3}<0\right)$ the pulse undergoes temporal and transverse compression. Indeed, in that case Eq. (4) is the 3D [i.e., $(x, y, t)$ ] NLS, which is known to have solutions that become singular in finite distance $z$ [2]. The dynamics in the case of normal time dispersion $\left(\epsilon_{3}>0\right)$ is much more complicated, however, because of the opposite signs of diffraction and time dispersion. The question whether small normal time dispersion can arrest singularity formation has defied research efforts for many years. In $[17,19]$ a reduced ordinary differential equation for the evolution of the pulse width at $t_{m}$, the time of the initial peak, was derived which showed that small normal time dispersion arrests the collapse at $t_{m}$. As a result, the pulse splits into two temporal peaks which continue to focus. In [17] it was conjectured that the new peaks would continue to split into "progressively smaller scale" and therefore that small normal time dispersion arrests self-focusing through multiple splitting. Numerical simulations of Eq. (4) $[19-21,6,22,14]$ showed that self-focusing of the two peaks leads to the formation of temporal shocks at the peak edges (see Fig. 1). As a result, in all the above studies, which used "standard" numerical methods, the simulations could not go beyond the shock formation and were thus unable to determine whether secondary splittings occur and whether the solution ultimately becomes singular. In [6] a reduced system of PDE's for self-focusing in Eq. (4) was derived which is valid for all $t$ cross sections. Analysis of the reduced system showed that, while self-focusing is arrested in the near vicinity of $t_{m}$, it continues elsewhere. However, the validity of the reduced system breaks down as the shock edges of the two peaks form. Therefore, one cannot use the reduced system to predict whether multiple splitting would occur and/or whether the solution ultimately becomes singular. Analysis of the reduced system did reveal, however, that temporal splitting is associated with the transition from independent 2D collapse of each $t$ cross section to full 3D dynamics. Therefore, it was suggested in [6] that the two peaks would not necessarily split again.

Temporal splitting of ultrashort pulses was observed experimentally in $[22,14]$. In these experiments secondary splittings were also observed at even higher input powers. Nevertheless, these observations do not imply that solutions of Eq. (4) undergo multiple splittings, because these secondary splittings were observed at such high powers where the validity of Eq. (4) breaks down, as additional physical mechanisms become important [13,15,23]. Recently, Germaschewski et al. [16] used an adaptive mesh refinement method to solve Eq. (4) beyond the pulse splitting. These simulations show that after the pulse splitting the two peaks continue to self-focus, resulting in the formation of temporal shocks. The two peaks do not undergo a similar secondary splitting. Rather, they "decay into secondary structures appearing in the outer parts of the far edges while they disperse." Similar results were also obtained by Coleman and Sulem using a dynamic mesh refinement method [24].

\section{A. Results}

In Figures 1-3 we present simulations of the timedispersive NLS (4) performed using the IGR method, with $\epsilon_{3}=1 / 32$ and the initial conditions

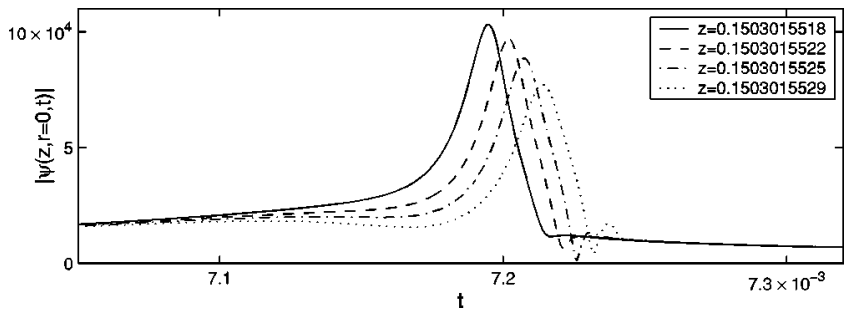

FIG. 10. Enlarged view of $|\psi(z, r=0, t)|$ for the simulation of Fig. 9, showing decay of maximum amplitude and emergence of small structures. 


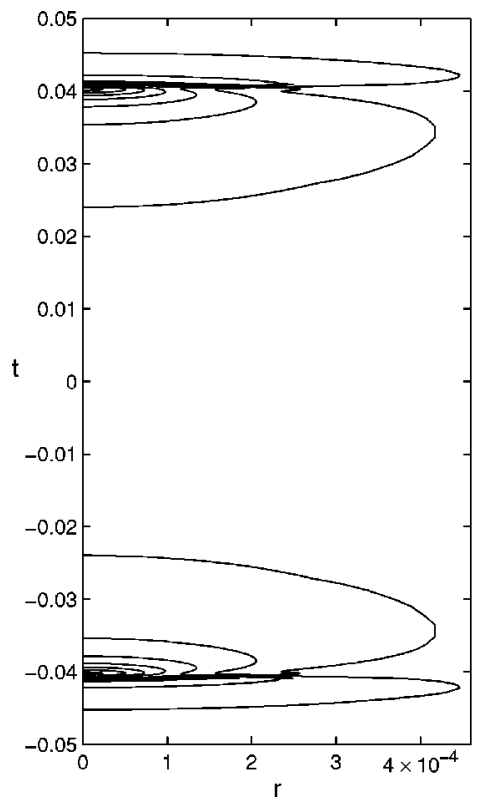

$$
\psi_{0}=A \sqrt{2} \exp \left(-\frac{x^{2}+y^{2}}{2}-t^{2}\right)
$$

We use the values of $A=1.75, A=2$, and $A=3$, whose peak power at $t=0$ is $1.65 P_{c}, 2.15 P_{c}$, and $4.83 P_{c}$, respectively, where $P_{c}$ is the threshold power for collapse [1]. In these simulations we integrate the equation on a domain $[r, t]$ $=[0,4] \times[0,12 / \sqrt{32}]$ with an initial uniform mesh of 100 $\times 300$. With the IGR we reached the smallest mesh size of $\delta r=2.1 \times 10^{-3}, \delta t=1.4863 \times 10^{-4}$ for the simulation of Fig. $1, \delta r=1.0197 \times 10^{-4}, \delta t=6.4876 \times 10^{-6}$ for Fig. 2, and $\delta r=1.911 \times 10^{-6}, \delta t=4.048 \times 10^{-7}$ for Fig. 3. We also plot the solution in the computational temporal variable (Figs. 1-3, right columns) to show that there is enough resolution in the oscillatory and shocklike regions. Indeed, even
FIG. 11. Contour plot of $\mid \psi(z$ $=0.1503015529, r, t) \mid$ for the simulation of Fig. 10, in physical variables (left) and computational variables (right).
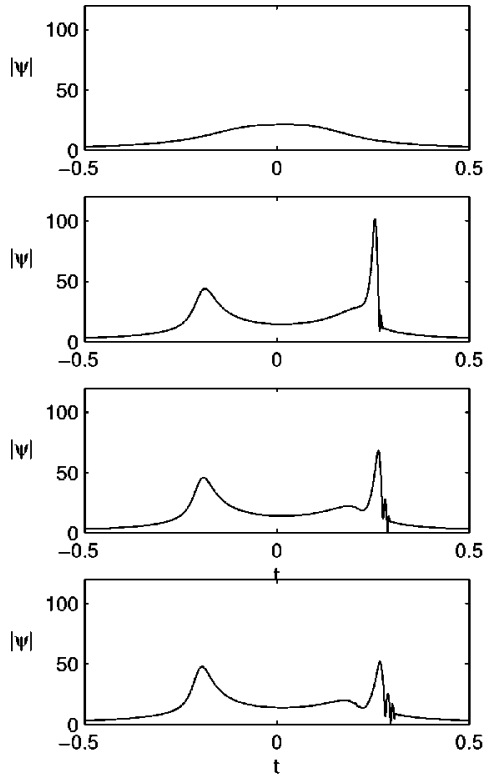
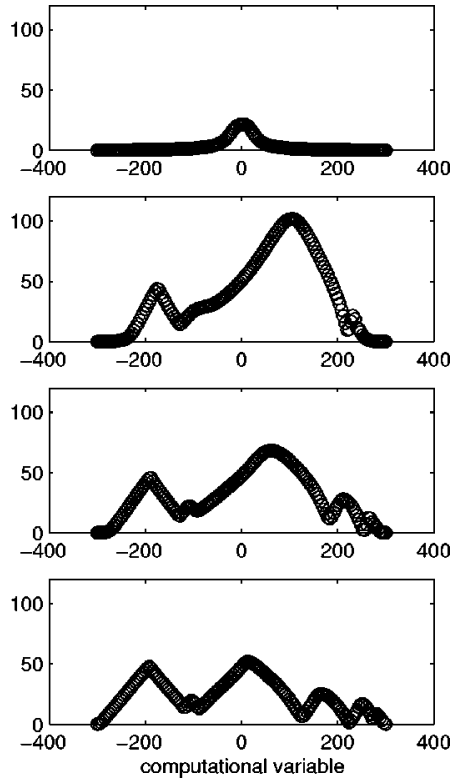

FIG. 12. Same as Fig. 1 for the solution of Eq. (3). 


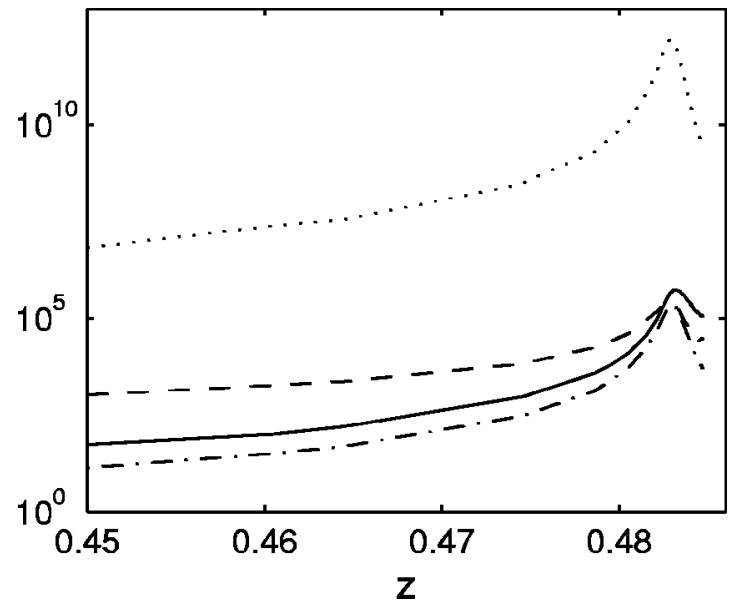

FIG. 13. $\max _{x, y, t}\left|\epsilon_{3} \psi_{t t}\right|$ (solid), $\max _{x, y, t}\left|\Delta_{\perp} \psi\right|$ (dots), $\left.\max _{x, y, t}\left|\Delta_{\perp} \psi+\right| \psi\right|^{2} \psi \mid$ (dashes), and $\max _{x, y, t}\left|\epsilon_{2}\left[\left(|\psi|^{2} \psi\right)_{t}-\Delta_{\perp} \psi t\right]\right|$ (dashdots), for the simulation of Fig. 12.

dispersion, diffraction, and nonlinearity (see Fig. 7), revealing the following dynamics. Initially time dispersion is negligible, i.e.,

$$
\epsilon_{3} \psi_{t t} \ll \Delta_{\perp} \psi+|\psi|^{2} \psi,
$$

and each $t$ cross section [i.e., the transverse $(x, y)$ plane for a given $t$ ] self-focuses independently according to Eq. (1). As diffraction and nonlinearity become larger they nearly balance each other [1]. As a result, the time dispersion becomes comparable to the balance of diffraction and nonlinearity while still being small compared with each of them separately, i.e.,

$$
\epsilon_{3} \psi_{t t} \approx \Delta_{\perp} \psi+|\psi|^{2} \psi, \quad \epsilon_{3} \psi_{t t} \ll \Delta_{\perp} \psi,|\psi|^{2} \psi,
$$

and it leads to the arrest of collapse followed by temporal splitting [6]. As the two new peaks self-focus and develop shock edges, the time dispersion becomes larger than the balance of diffraction and nonlinearity, i.e.,

$$
\epsilon_{3} \psi_{t t} \gg \Delta_{\perp} \psi+|\psi|^{2} \psi
$$

Therefore, the postshock dynamics is dominated, to leading order, by the $1 D$ linear Schrödinger equation $i \psi_{z}-\epsilon_{3} \psi_{t t}$ $=0$, and the pulse undergoes temporal dispersion, which leads to the disintegration of the shocks and to temporal oscillations near the shocks. Note that at this stage the crosssectional power is above critical (see Fig. 8). This does not imply, however, that the pulse would self-focus radially, since this conclusion would hold when the dominant mechanisms are nonlinearity and diffraction, whereas at this stage the dominant mechanism is temporal dispersion, which leads to temporal spreading. Ultimately, the power of all $t$ cross sections goes below critical and the amplitude of the pulse decays to zero, as diffraction, dispersion, and nonlinearity are all of equal magnitude.

We recall that in [17] Zharova et al. solved Eq. (4) with $\epsilon_{3}=1$, and the initial condition $\psi_{0}=4 \exp \left[-\left(x^{2}+y^{2}\right) / 2\right.$ $\left.-t^{2} / 32\right]$, whose peak power at $t=0$ is $4.3 P_{c}$. In their simulations the temporal splitting was followed by a secondary splitting, leading Zharova et al. to the conjecture that small normal time dispersion arrests collapse through multiple splittings. Although it has been suspected that these secondary splittings were a numerical artifact due to underresolution, this specific problem turned out to be computationally hard. In fact, even Germaschewski et al. [16] could not continue the simulation of the "Zharova problem" beyond the pulse splitting, due to under-resolution. In Figs. 9-11 we present simulations of the Zharova problem that go beyond the pulse splitting. Our simulations reveal a dynamics similar to the one observed earlier. In particular, there are no secondary splittings. Note that in the computational variables the solution remains relatively smooth even after the pulse splitting (Fig. 11).

\section{FULL MODEL}

The insight gained from the simulations of Eq. (4) is useful for understanding the self-focusing dynamics in the more comprehensive physical model (3). In Fig. 12 we present simulations of Eq. (3) where $\epsilon_{3}=1 / 32$ and $\epsilon_{2}=0.001 / \sqrt{32}$. The initial condition is Eq. (10) with $A=1.75$. We integrate on a domain $[r, t]=[0,4] \times[-12 / \sqrt{32}, 12 / \sqrt{32}]$ with an initial uniform grid of $100 \times 600$. Because the solution of Eq. (3) is not symmetric in $t$, the number of grid points in the $t$ variable is twice the number in our simulations of Eq. (4).

The results of the simulation confirm the theoretical prediction made in [8] that the pulse splits in an asymmetric fashion, with the front peak being lower than the rear peak, and also the observation first made in [13] that the (higher) rear peak becomes lower than the front peak with further propagation. Our simulations reveal that the arrest of collapse of the rear peak is done in an oscillatory manner which is reminiscent of the symmetric case (4). Note that the solution in the rescaled variables remains reasonably smooth, even when the physical solution develops very steep gradients (Fig. 12).

When we plot the relative magnitudes of the various mechanisms we see that, as in the case of the time-dispersive NLS (4), after the asymmetric pulse splitting linear time dispersion becomes the dominant mechanism (see Fig. 13). The magnitude of the $\epsilon_{2}$ symmetry-breaking terms becomes comparable to the sum of diffraction and nonlinearity just after the pulse splitting, but subsequently becomes small again. We thus conclude that these symmetry-breaking terms have an important effect only during the pulse splitting event. Note, however, that the last conclusion is valid when $\epsilon_{2}$ $\ll \epsilon_{3}$, whereas for longer pulses $\epsilon_{2}$ can be larger than both $\epsilon_{3}$ and $\epsilon_{1}[8]$.

\section{FINAL REMARK}

In this study we showed that the IGR method can be used to solve time-dispersive NLS equations with near- 
singularities and shocks. The IGR method gives more control of the grid distributions (by grid mapping iteration) than other moving mesh methods. In addition, it is easier to implement than local refinement methods. Indeed, the IGR method allowed us to solve the Zharova problem beyond the pulse splitting. We believe, therefore, that the IGR method can be successfully applied to other problems in nonlinear optics where singularities or shocks develop.

\section{ACKNOWLEDGMENTS}

This work is supported in part through the Research Grant Council of Hong Kong by Grant Nos. HKUST 6165/97P and HKUST 6176/99P. The work of G.F. is also partially supported by Grant No. 2000311 from the United StatesIsrael Binational Science Foundation (BSF), Jerusalem, Israel.
[1] G. Fibich and G.C. Papanicolaou, SIAM (Soc. Ind. Appl. Math.) J. Appl. Math. 60, 183 (1999).

[2] C. Sulem and P.L. Sulem, The Nonlinear Schrödinger Equation (Springer, New York, 1999).

[3] G. Fibich and B. Ilan, Appl. Numer. Math. 44, 63 (2003).

[4] D.W. McLaughlin, G.C. Papanicolaou, C. Sulem, and P. L. Sulem, Phys. Rev. A 34, 1200 (1986).

[5] M. J. Landman, G.C. Papanicolaou, C. Sulem, P.L. Sulem, and X.P. Wang, Physica D 47, 393 (1991).

[6] G. Fibich, V.M. Malkin, and G.C. Papanicolaou, Phys. Rev. A 52, 4218 (1995).

[7] W. Ren and X.P. Wang, J. Comput. Phys. 159, 246 (2000).

[8] G. Fibich and G.C. Papanicolaou, Opt. Lett. 22, 1379 (1997).

[9] S. Chi and Q. Guo, Opt. Lett. 20, 1598 (1995).

[10] G. Fibich and B. Ilan, Physica D 157, 112 (2001).

[11] G. Fibich and B. Ilan, Opt. Lett. 26, 840 (2001).

[12] G. Fibich and G.C. Papanicolaou, Phys. Lett. A 239, 167 (1998).
[13] J.K. Ranka and A.L. Gaeta, Opt. Lett. 23, 534 (1998).

[14] S.A. Diddams, H.K. Eaton, A.A. Zozulya, and T.S. Clement, Opt. Lett. 23, 379 (1998).

[15] A.A. Zozulya, S.A. Diddams, and T.S. Clement, Phys. Rev. A 58, 3303 (1998).

[16] K. Germaschewski, R. Grauer, L. Berge, V.K. Mezentsev, and J.J. Rasmussen, Physica D 151, 175 (2001).

[17] N.A. Zharova, A.G. Litvak, T.A. Petrova, A.M. Sergeev, and A.D. Yunakovsky, JETP Lett. 44, 13 (1986).

[18] J.U. Brackbill and J.S. Saltzman, J. Comput. Phys. 46, 342 (1982).

[19] G.G. Luther, A.C. Newell, and J.V. Moloney, Physica D 74, 59 (1994).

[20] J.E. Rothenberg, Opt. Lett. 17, 583 (1992).

[21] P. Chernev and V. Petrov, Opt. Lett. 17, 172 (1992).

[22] J.K. Ranka, R.W. Schirmer, and A.L. Gaeta, Phys. Rev. Lett. 77, 3783 (1996).

[23] A.L. Gaeta, Phys. Rev. Lett. 84, 3582 (2000).

[24] J. Coleman and C. Sulem, Phys. Rev. E 66, 036701 (2002). 